Ophthalmologe 2009 · 106:297-298

DOI 10.1007/s00347-008-1868-y

Online publiziert: 29. März 2009

๑) Springer Medizin Verlag 2009

0. Strauß

Experimentelle Ophthalmologie, Klinik und Poliklinik für Augenheilkunde, Klinikum der Universität Regensburg

\title{
Das retinale Pigmentepithel
}

Formen der Photorezeptordegenerationen und damit der Blindheit dar.

Die Aufgaben des retinalen Pigmentepithels in dieser Funktionseinheit bestehen in homöostatischen Funktionen und in Funktionen, die direkt mit Lichtwahrnehmung in Verbindung stehen [7]. Eine Pigmentepithelzelle interagiert hierbei mit ca. 23 Zapfen in der Fovea. Durch die Lichtabsorption wird das retinale Pigmentepithel in der Tat auf Temperaturen von über $40^{\circ} \mathrm{C}$ aufgeheizt [4]. Der Abtransport der Wärme geschieht wahrscheinlich über die Durchblutung der Chorioidea, die eine höhere Durchblutung als die Nierenrinde aufweist. Die Funktionseinheit mit den Photorezeptoren stellt das Pigmentepithel durch transepithelialen Transport von Wasser, Ionen und Nährstoffen [3], durch die Kontrolle der Ionenzusammensetzung im subretinalen Raum [6], durch den Retinalzyklus, durch die Phagozytose abgestoßener Spitzen der Außensegmente der Photorezeptoren [10] und durch seine sekretorischen Funktionen her [5]. Darüber hinaus ist das Pigmentepithel aktiv an der Entstehung des immunprivilegierten intraokulären Raums durch Immunsuppression beteiligt [8]. Der Ausfall einer einzelnen aus fast allen diesen Funktionen führt zur Degeneration der Photorezeptoren.

In hereditären Formen der Retinadegeneration (s. Beitrag Preising und Lorenz) sind die Funktionen des Retinalzyklus und die Phagozytoseregulation vorrangig betroffen. Beispiele sind hier das RPE65-Gen, dessen Genprodukt eine zentrale Rolle im Zyklus des Retinals zwischen seinen Isomeren all-trans und 11-cis spielt [11], oder das MerTK-Gen [2], das für einen membranständigen Rezeptor kodiert, der für die Initiierung der Phagozytose von großer Bedeutung ist.

In der Klinik können Degenerationen der Retina sowohl durch Zerstörung des retinalen Pigmentepithels als auch durch eine aktive Rolle des retinalen Pigmentepithels entstehen (s. Beitrag Gamules$\mathrm{cu}$, Renner und Helbig). Alterskorrelierte Makulopathie oder auch die alterskorrelierte Makuladegeneration gehen auf eine mit dem Alter fortschreitende Funktionsminderung und später auf Pigmentzellverlust zurück [1]. Auf der anderen Seite kann das retinale Pigmentepithel (RPE) aktiv an der Entstehung von Erkrankungen beteiligt sein, indem es $\mathrm{zu}$ Fehlregulationen der ansonsten "guten“ Funktionen kommt [9]. Bei der proliferativen Vitreoretinopathie (PVR) kommt es zur Proliferation von Pigmentepithelzellen und bei Neovaskularisationen zu pathologischen Veränderungen der sekretorischen Aktivität. Diese aktiven Beteiligungen lassen sich aber auch klinisch nutzen, indem z. B. die Sekretion protektiver Faktoren oder immunmodulatorischer Faktoren angeregt werden kann.

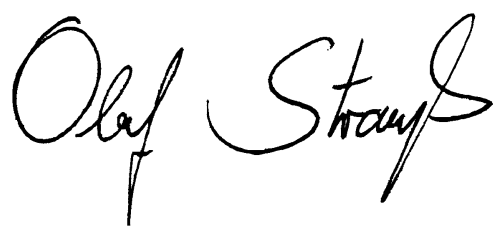

Olaf Strauß

\section{Korrespondenzadresse \\ Prof. Dr. 0. Strauß}

Experimentelle Ophthalmologie, Klinik und Poliklinik für Augenheilkunde, Klinikum der Universität Regensburg Franz-Josef-Str.aße11, 93053 Regensburg strauss@eye-regensburg.de de auf der Seite des retinalen Pigmentepithels stellen die Ursachen für die häufigsten 
Interessenkonflikt. Der korrespondierende Autor gibt an, dass kein Interessenkonflikt besteht.

\section{Literatur}

1. Boulton M (1991) Ageing of the retinal pigment epithelium. In: Osborne NN, Chader GJ (eds) Progress in retinal research. Pergamon Press, Oxford New York, p 125-151

2. Gal A, Li Y, Thompson DA et al (2000) Mutations in MERTK, the human orthologue of the RCS rat retinal dystrophy gene, cause retinitis pigmentosa. Nat Genet 26:270-271

3. Hughes BA, Gallemore RP, Miller SS (1998) Transport mechanisms in the retinal pigment epithelium. In: Marmor MF, Wolfensberger TJ (eds) The retinal pigment epithelium. Oxford University Press, New York Oxford, p 103-134

4. Parver LM, Auker C, Carpenter DO (1980) Choroidal blood flow as a heat dissipating mechanism in the macula. Am J Ophthalmol 89:641-646

5. Rosenthal R, Strauss $\mathrm{O}$ (2002) $\mathrm{Ca}^{2+}$-channels in the RPE. Adv Exp Med Biol 514:225-235

6. Steinberg RH (1985) Interactions between the retinal pigment epithelium and the neural retina. Doc Ophthalmol 60:327-346

7. Strauss $O$ (2005) The retinal pigment epithelium in visual function. Physiol Rev 85:845-881

8. Streilein JW, Ma N, Wenkel H et al (2002) Immunobiology and privilege of neuronal retina and pigment epithelium transplants. Vision Res 42:487495

9. Tanihara H, Inatani M, Honda Y (1997) Growth factors and their receptors in the retina and pigment epithelium. Prog Retin Eye Res 16:271-301

10. Thompson DA, Gal A (2003) Vitamin A metabolism in the retinal pigment epithelium: genes, mutations and diseases. Prog Retin Eye Res 22:683-703

11. Xue L, Gollapalli DR, Maiti P et al (2004) A palmitoylation switch mechanism in the regulation of the visual cycle. Cell 117:761-771

\section{Günter K. Krieglstein (Hrsg.) Glaukom 2008}

Eine moderne Glaukom-Sprechstunde

Berlin, Heidelberg, New York: Springer Verlag 2008, (ISBN 978-3-540-87893-3), 9.00 EUR

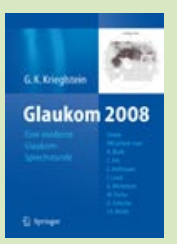

Der Springer Verlag hat ein neues Buch aufgelegt, welches sich an Glaukominteressierte richtet. Am 18/19. April 2008 trafen sich unter Leitung von Herrn

Prof. Krieglstein (Köln) namhafte Glaukomexperten in Niedernberg, um anhand von Fallbeispielen eine moderne Glaukomsprechstunde zu gestalten. Diagnostik und Therapie von Glaukompatienten unterliegt einem Wandel, der auch von neuen Untersuchungsmethoden herrührt. Um diesen Neuerungen gerecht zu werden wurde diese moderne Glaukomsprechstunde zusammengestellt. Hierbei wurde von den Referenten eine Serie von Patientenvorstellungen ausgewählt, um die Fortschritte in der Diagnostik zu diskutieren. Dabei wurden die Schwerpunkte Risikoabschätzung, Progression, morphologische und sinnesphysiologische Diagnostik sowie spezielle Problemstellungen berücksichtigt und erklärt.

Das Buch enthält 175 Seiten auf denen 253 Abbildungen Platz finden. Das allein zeigt schon den hohen Anschauungsgrad und die Gewichtung des Buches. Damit lässt sich das Buch sehr gut lesen, oder besser noch anschauen. Moderne Glaukomdiagnostik ist moderne Bildgebung, die in die klinischen Entscheidungen Einfluss findet. So sind jedem Fall mit einer kurzen Vorstellung zur Krankengeschichte zahlreiche Abbildungen zur Seite gestellt, die helfen den Fall zu bewerten und die klinisch/therapeutischen Entscheidungen nachzuvollziehen. Unter dem Teil „Risikoaspekte“ finden sich Abbildungen zu Tagesdruckprofilen, die deutlich machen, wie wichtig diese bei der klinischen Entscheidungsfindung sind. Der Einsatz von GDX und HRT wird beispielgebend dargestellt; besondere Bewertung findet der Aspekt der Progression, die exemplarisch durch die modernen Bildgebungen (inklusive Progressionsanalysen) gezeigt wird. Klar zur Geltung kommen die Korrelationen von HRT und
Papillenfotos im nächsten Teil ",Morphologische Aspekte". Spezielle Diagnostik, wie die 24-Stunden-Blutdruckmessung und die Frequenzverdopplungsperimetrie, und spezielle Fragestellungen (MRT und Neuroimaging) runden das Bild ab.

Bemerkenswert ist die gute Qualität der Abbildungen und die klare Textsprache. So ist dieses Buch nicht nur für Glaukomexperten und -interessierte sondern auch für Fachärzte gleichermaßen geeignet und empfehlenswert.

PD Dr. H. Thieme, Universitäts-Augenklinik Mainz 